\title{
Optomechanical Design for Precision Lens Positioning and Mounting
}

\author{
Shuping Wang ${ }^{*}$, Chi Zhang2, Mark Alt ${ }^{2}$, Colleen Davis ${ }^{2}$, Michael L. Gardner ${ }^{2}$, Zheng Ji ${ }^{3}$ \\ ${ }^{1}$ College of Engineering, University of North Texas, Denton, TX, USA \\ ${ }^{2}$ Sanmina Corporation, Carrollton, TX, USA \\ ${ }^{3}$ Bosch Automotive Products Co., Ltd., Suzhou, China \\ Email: *shuping@unt.edu
}

Received 15 March 2016; accepted 19 August 2016; published 25 August 2016

\begin{abstract}
The proper choice of optomechanical components along with alignment techniques is vital to the successful implementation of lens positioning and mounting. In general, there is a trade-off between lens positioning accuracy and manufacturing cost. In order to achieve optimal precisionto-cost ratio, a careful optomechanical design together with accurate alignment and assembly is necessary. This paper presents a lens positioning and mounting in a few microns accuracy using a kinematic mount module. The optomechanical design of the kinematic mount module and the associated fixtures based on the simulation study of components sensitivity are presented. The processes for alignment and assembly, which separate decenter from tip-tilt, are developed and presented. Finally, the prototypes are created and testing results are presented. The optomechanical design that allows the separation of decenter from tip-tilt considerably simplifies the positioning and assembly process and reduces the cost for mass production.
\end{abstract}

\section{Keywords}

Optomechanical Design, Lens Mount, Lens Positioning

\section{Introduction}

High-precision positioning and mounting individual optical lenses in optical instruments are critical for higher-performance applications. For many instruments with compact arrangement, the room for mechanical equipment to align an individual optical lens in the end product is very limited. Thus manufacturing becomes more cost and time efficient when modular design is used where the lens alignment and subassembly are achieved at the module level with separate setups [1]. The finished module should be easily integrated into the instruments. This paper presents an overview of the efforts on prototype development of a kinematic mount module for high-precision lens assembly. The module consists of two major parts: the fixed kinematic mount base and the floating kinematic lens unit. The fixed kinematic mount base defines its position relative to the Optical Axis (OA) of the instrument. It is designed to be easily plugged into the instrument. The floating kinematic lens unit

${ }^{*}$ Corresponding author.

How to cite this paper: Wang, S.P., Zhang, C., Alt, M., Davis, C., Gardner, M.L. and Ji, Z. (2016) Optomechanical Design for Precision Lens Positioning and Mounting. Optics and Photonics Journal, 6, 1-7.

http://dx.doi.org/10.4236/opj.2016.68B001 
that holds the lens is aligned with the OA and attached to the fixed kinematic mount base. The finished module is integrated into the instrument as follows: plug the fixed kinematic mount base into the instrument; the floating kinematic lens unit is then mounted into the fixed kinematic base and held in position by engaging air pressure on a pneumatic cylinder. The instrument system requires the plano-convex lens to be aligned with and positioned on the system OA. A tolerance of $\pm 3 \mu \mathrm{m}$ apex decenter from the OA and $\pm 0.1^{\circ}$ tilt angle of the planosurface normal away from the OA is necessary. Therefore, common types of lower-precision techniques, such as simple spring clips or burnished cell mounting, are not sufficient [2]. Active optical alignment and specially designed mechanical fixtures (i.e. kinematic constraining each of the six degrees of freedom) are needed to achieve the highest precision of the lens position. The rest of the paper is organized as follows. Section 2 briefly reviews the optical alignment system design and simulations. Section 3 presents the optomechanical design of the kinematic mount module and process development for the prototype fabrication. Section 4 introduces the testing results and analysis, followed by the summaries in Section 5 .

\section{Review of Optical Alignment System Design and Simulation}

The objective of the project is to align and mount a plano-convex ball lens (hereafter called working lens), as shown in Figure 1, to an instrument so that the apex of the working lens is on the system OA with a tolerance of $\pm 3 \mu \mathrm{m}$ in $\mathrm{X}$ and $\mathrm{Y}$ directions and the tilt angle of the plano-surface normal away from the OA is less than $0.1^{\circ}$. The apex is defined as the point on the convex surface where its tangent plane is parallel to the plano surface of the working lens. The working lens is made of BK7 glass, therefore is transparent to visible light. The following concept has been used to identify whether the apex of the working lens is located on the OA. A collimated beam of light that defines the OA (e.g. $632 \mathrm{~nm}$ HeNe laser) enters the plano surface of the working lens perpendicularly, as long as the transmitted light is focused on the optical axis, the apex of the working lens is guaranteed on the optical axis.

Based on the above concept, an alignment strategy that separates tip-tilt tuning from decenter alignment has been developed. The strategy makes the alignment system more manufacturable which is especially vital in light of the future automation production systems. Specifically, the plano surface of the working lens is aligned using two degrees of freedom $(\theta$ and $\varphi$ ) followed by the alignment of apex of the working lens using three degrees of freedom ( $\mathrm{x}, \mathrm{y}$, and $\mathrm{z}$ ). Because the ball lens features very short back focal length, directly measuring the focal position of the incident beam is not practical for production setup. A recollimating lens is designed to be positioned after the back focal plane of the working lens; the defocused light is then recollimated. Thus a position detector can be placed some convenient distance from the recollimating lens to measure the position of the transmitted light as shown in Figure 2 (position detector is not shown). The recollimating lens is designed to be a plano-convex aspheric lens as detailed in [3]; its plano surface plays a role during the system calibration process that will be explained later.

Simulation has been conducted, as detailed in [3], to study sensitivity of the alignment system to position of the apex of the working lens and tilt angle of the plano surface normal to the OA. Specifications for optome-

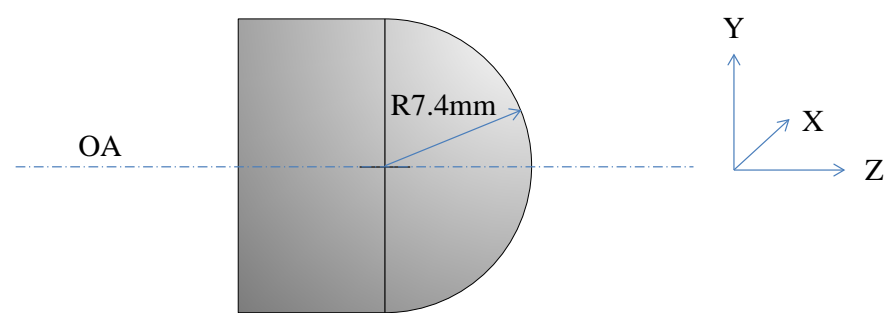

Figure 1. The schematic of the plano-convex lens (working lens).

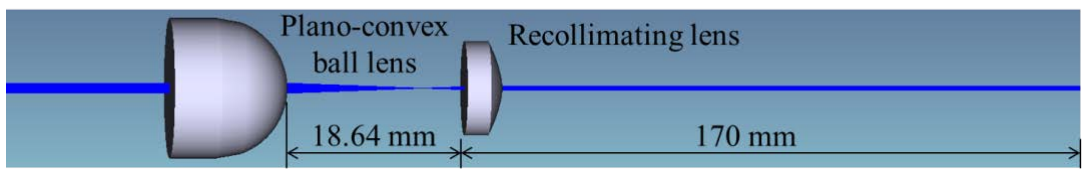

Figure 2. The defocused light is collimated by the recollimating lens. 
chanical fixtures used for both alignment and prototyping are also obtained through the simulation. A beam scanner (of submicron resolution) is proposed for position detection in the alignment system. It is important to understand the smallest potential deviation of the working lens that will register on the beam scanner in both tilt and decentering. The beam shift, caused by the deviation of the working lens, on the beam scanner depends on the distance between the recollimating lens and the beam scanner. Proper distances between the working lens and the recollimating lens and that between the recollimating lens and the position detector are determined by simulation and labeled in Figure 2. The tilt angle of the working lens is monitored using an autocollimator (resolution of 0.01 arc-second) with its output beam aligned to the OA of the alignment system. The plano surface of the working lens (uncoated) is used as the external reflector of the autocollimator.

\section{Optomechanical Design and Prototype Fabrication Process}

\subsection{Optomechanical Design}

The optomechanical system consists of several fixtures: fixed kinematic mount base, floating kinematic lens unit that holds the working lens, He-Ne laser source fixture, autocollimator fixture, and beam scanner fixture as shown in Figure 3. This paper mainly focus on discussing the design and prototyping of two major fixtures: the fixed kinematic mount base and the floating kinematic lens unit.

\subsubsection{Fixed Kinematic Mount Base}

The fixed kinematic mount base, shown in Figure 4, is to hold the floating kinematic lens unit and to establish its relationship to the system OA (He-Ne laser beam path). Thus the finished kinematic mount module can

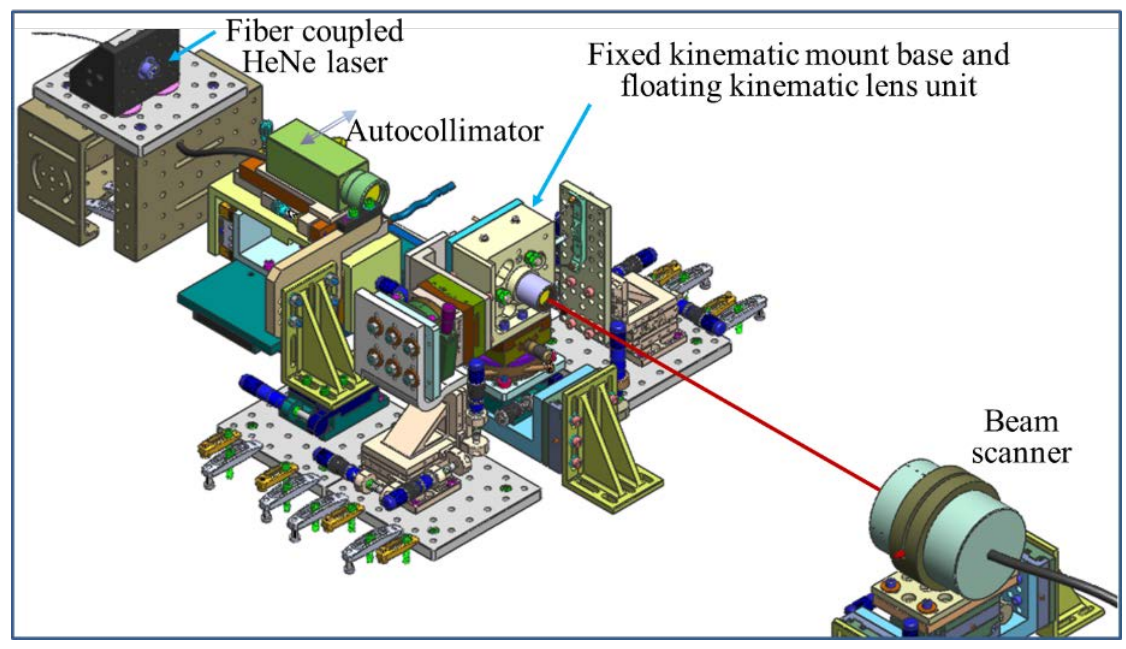

Figure 3. The schematic of the optomechanical system.

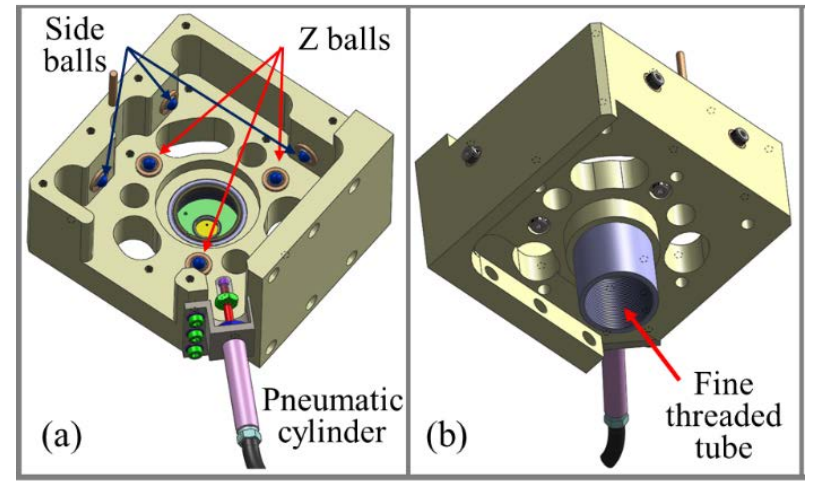

Figure 4. The schematic of the fixed kinematic mount base. (a) Front view; (b) Back view. 
achieve alignment-free installation into the next level instrument as well as to meet a tolerance of $\pm 3 \mu \mathrm{m}$ of the lens apex decenter from the $\mathrm{OA}$ and $0.1^{\circ}$ tilt angle of the plano-surface normal away from the OA.

The fixed kinematic mount base is therefore designed to sit on a five-axis stage, so its X-Y-Z position and tiptilt relative to the system OA can be fully adjusted. As part of the fixed kinematic mount base, a fine threaded tube is designed to hold the recollimating lens. The plano surface of the recollimating lens is uncoated and used as the reference for the autocollimator to measure the tiltangle of the fixed kinematic mount base. The He-Ne throughput beam is used together with a beam profiler to align the center of the fixed kinematic mount base to the system OA. To make such a design work, a removable lens needs to be added into the lens tube to create a well-collimated beam.

Inside the fixed kinematic mount base, a pneumatic cylinder engaged by air pressure is designed to hold the floating kinematic lens unit at different assembly stages. Three stainless steel Z-ball adjusters, with fine 0.20 $\mathrm{mm}$ per resolution thread, are used to define a reference plane to support the floating kinematiclens unit. Three similar side-ball adjusters are used to define X-Y position of the floating kinematiclens unit.

\subsubsection{Floating Kinematic Lens Unit}

To reduce the complexity of the lens alignment especially for mass production, the floating kinematic lens unit is specially designed to enable the separate movement in tip-tilt and X-Y position of the lens. The lens is attached and fixed onto a spherical gimbal plate by laser welding (by epoxy for prototyping) as shown in Figure 5(a). Tip-tilt plate, with the spherical feature at same dimension as the gimbal plate, is designed to host the gimbal plate in tight contact. Tip-tilt alignment of the lens is achieved by moving the gimbal plate against the tip-tilt plate. The floating kinematic lens unit is attained by laser welding the two parts together after alignment (using epoxy for prototype). Three surface-screw holes in gimbal plate are designed for gimbal (tip-tilt) adjustment fixture attachment. Two side-screw holes in tip-tilt plate enable the attachment of the X-Y alignment tool fixture.

An XY plate, as shown in Figure 5(b), is designed to provide a reference plane for X-Y alignment of the lens. The XY plate is supported by the Z-ball adjusters and held by the pneumatic cylinder against the side-ball adjusters inside the fixed kinematic mount base as described previously. The XY plate possesses ware pads at all contact positions with the ball adjusters. $\mathrm{X}-\mathrm{Y}$ alignment is achieved by sliding the tip-tilt plate subassembly that is in tight contact with the $\mathrm{XY}$ plate. After completing the $\mathrm{X}$-Y alignment, the tip-tilt plate subassembly and the $\mathrm{XY}$ plate are laser welded together (using epoxy for prototype) to produce the floating kinematic lens unit.

\subsection{Process Development for Prototype Fabrication}

To optimize the performance of manufacture system, the following assembly process for prototype is developed.

1) System Calibration

System calibration is accomplished in two steps for each kinematic mount module assembly. A five-axis stage that holds the fixed kinematic mount unit is calibrated first followed by calibration of the Z-ball and side-ball adjusters. The recollimating lens combined with an over-the-counter lens inside the fixed tube as shown in Figure 6(a) (lens pair) is used to align the center of the fixed kinematic mount unit to the OA via the five-axis stage. The positions of the five axes are then fixed and maintained throughout the entire assembly process. A calibration plate as shown in Figure 6(b) is used to define positions of the Z-ball and the side-ball adjusters. Tip-tilt of

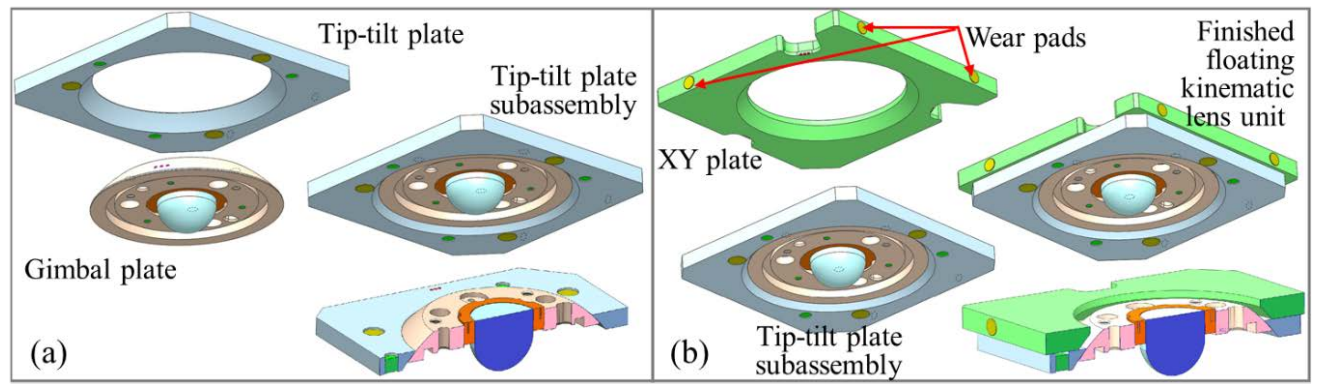

Figure 5. The schematic of the floating kinematic lens unit. (a) Gimbal plate (with lens) placed into the tip-tilt plate; (b) Tip-tilt subassembly attached on the XY plate. 


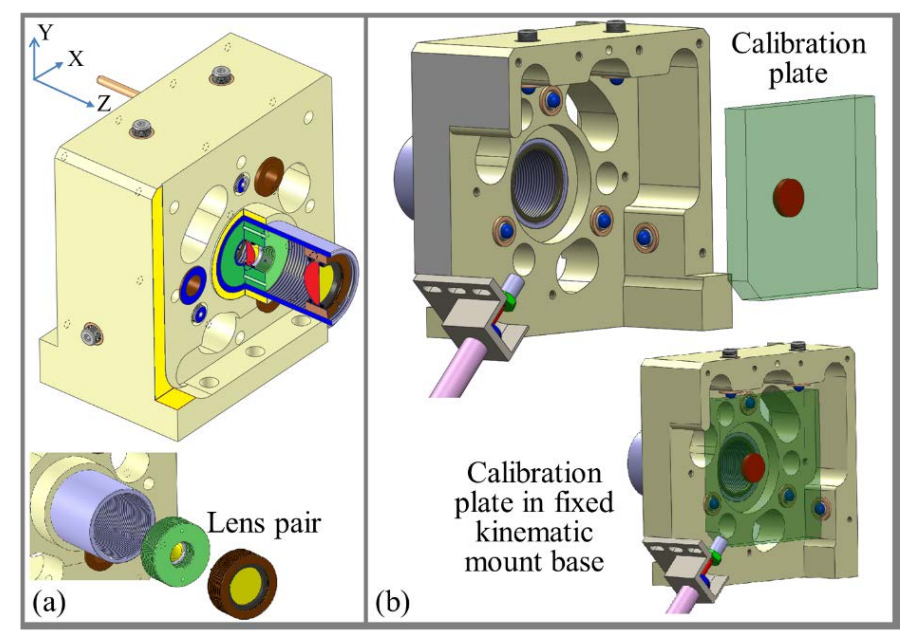

Figure 6. Calibration plate in fixed kinematic mount. (a) A lens pair in the fixed tube is used to align the center of the fixed kinematic mount base to the OA; (b) A calibration plate is used to define positions of the Z-ball and the side-ball adjusters.

the calibration plate is adjusted by the Z-ball adjusters and monitored by an autocollimator. X-Y positions of the calibration plate are adjusted by the side-ball adjusters and monitored by a beam scanner. After calibration the Z-ball and the side-ball adjusters in the fixed kinematic mount base are all fixed by UV/thermal dual cure epoxy to ensure no movement during the entire assembly process.

2) Tip-Tilt Alignment

The working lens attached to a solder sleeve is tightly fitted into a spherical gimbal plate using UV curable epoxy (Dymax OP-24-Rev B) for prototype. The spherical gimbal plate is then placed into the tip-tilt plate with a thin layer of epoxy in between. Both plates are then assembled into a specially designed holder fixture, which is threaded onto the fixed kinematic mount base tightly as shown in Figure 7. The tip-tilt alignment is conducted by adjusting the gimbal plate grabber that is attached on a five-axis stage. The autocollimator is brought into the system optical path to measure tilt angle. After achieving the tip-tilt alignment, pre-applied epoxy is UV cured to temporarily hold the alignment. Remove the gimbal adjustment fixture out of the fixed kinematic mount base and remove the gimbal plate subassembly from the holder fixture. Finally thermal cures the epoxy to fix the tip-tilt alignment permanently.

3) X-Y Alignment

Attach the $\mathrm{X}-\mathrm{Y}$ alignment fixture that is held by an $\mathrm{X}-\mathrm{Y}$ translation stage to the tip-tilt plate subassembly through the side screw holes as shown in Figure 8. Insert the tip-tilt plate subassembly together with the XY plate into the fixed kinematic mount base with epoxy applied to the interface of the two parts. The pneumatic cylinder is turned onto hold the XY plate, while a main clamp frame is attached to ensure the firm contact of the two parts during the alignment process. Prior to the X-Y alignment, the autocollimator is brought into optical path to double check the tip-tilt of the lens. Align X-Y position of the tip-tilt plate subassembly until the laser beam hits the preset zero position in the beam scanner. UV cures the floating kinematic lens unit assembly before it is removed from the fixed kinematic mount base. The finished assembly is then put into an oven for final thermal curing.

\section{Testing Results and Analysis}

Ten prototypes of kinematic mount module have been fabricated using the process described in section 3. Apex position accuracy and tip-tilt accuracy of the finished modules were tested. The same metrology system developed for the kinematic mount module assembly was used for testing. The testing system was calibrated by means of the same calibration plate used for module fabrications. The testing was conducted by aligning the center of one fixed kinematic mount base to the OA first. The testing system was then calibrated by loading the calibration plate into the fixed kinematic mount base and normalizing the plano-surface tip-tilt readings and the $\mathrm{X}-\mathrm{Y}$ position detector readings to zeroes. The tilt of the plano surface and the apex position of the lens, relative 


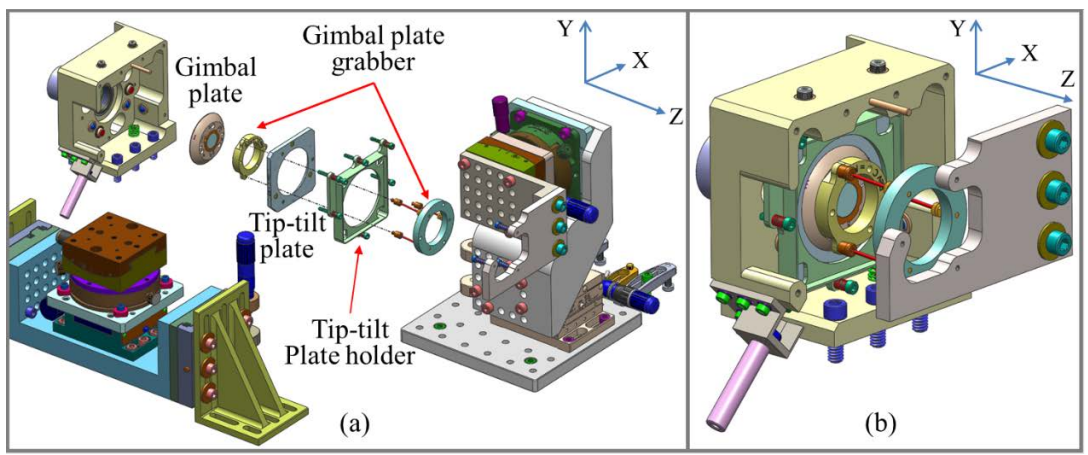

Figure 7. Tip-tilt alignment. (a) Explode view; (b) Detail view.

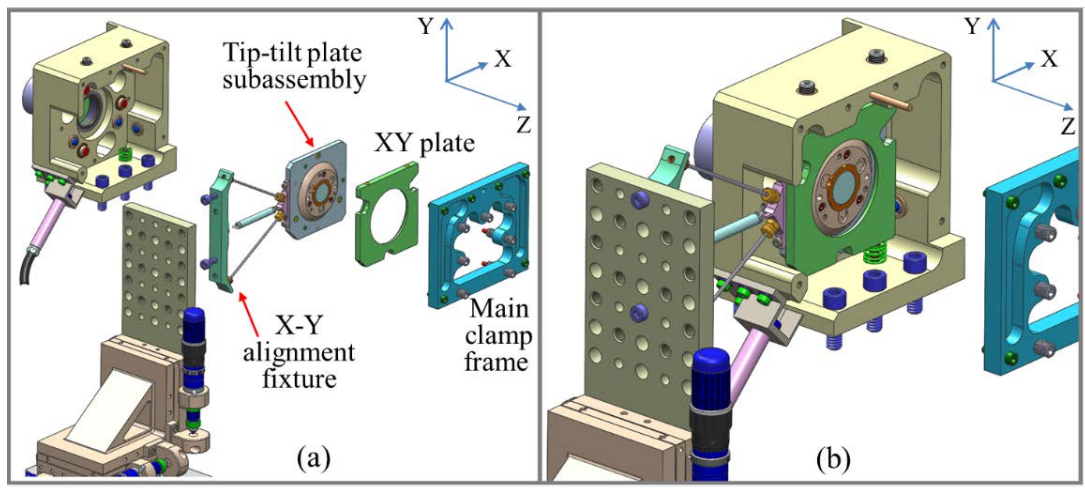

Figure 8. X-Y alignment. (a) Explode view; (b) Detail view.

to the calibration standard, were measured for each floating kinematic lens unit. Each time a complete set of 5 load/unload readings were recorded along with fresh calibration plate readings. The average of the five measured values for each floating kinematic lens unit was used as the assigned X-Y position and the tilt angle for each unit as shown in Figure 9(a) and Figure 9(b) respectively. The standard deviations of the X-Y position and the plano-surface tilt angle were less than $0.15 \mu \mathrm{m}$ and 13 arcSec respectively.

The tilt angle of the plano surface was measured directly using the autocollimator with its output beam aligned to the system OA. The result of Figure 9(b) indicates that the tilt angles for all samples were less than $0.1^{\circ}$, meeting the tip-tilt specifications. On the other hand, the apex positions $\mathrm{X}$ and $\mathrm{Y}$ shown in Figure 9(a) were derived from the values obtained by the position detector (i.e. the beam scanner). It is important to understand that the position detector measures the image position of the incident beam relative to the normalized image position (0, 0); $\mathrm{X}$ and $\mathrm{Y}$ represent apex position precisely only when tilt angles are zeros since both apex decenter and plano-surface tilt contribute to the image position shift. Therefore the $\mathrm{X}$ and $\mathrm{Y}$ positions presented in Figure 9(a) denote amount of image position shifts caused by the combination of apex decenter and the plano-surface tilt. Thus the actual apex decenter for each sample should be the $\mathrm{X}$ and $\mathrm{Y}$ values in Figure 9(a) plus/minus the image position shift caused by the plano-surface tilt shown in Figure 9(b). Based on the simulation, the maximum $\mathrm{X}$ and $\mathrm{Y}$ shift caused by the plano-surface tip-tilt was less than $0.2 \mu \mathrm{m}$ for the tilt angles shown in Figure 9(b). After considering the fact that the plano surface was not perfectly perpendicular to the incident beam as shown in Figure 9(b), the apex positions for all samples were still within the specified range, i.e. $\pm 3 \mu \mathrm{m}$.

To investigate the root cause of the errors, one floating kinematic lens unit was measured two more times after final UV/thermal cure of the unit. Each time a complete set of $10 \mathrm{load} / \mathrm{unload}$ readings were recorded along with fresh calibration plate readings. If the kinematic ball and wear pad mating were perfect, we would expect identical relative results between each set. However the repeatability was not perfect. A repeatability variation of about 0.3 to 0.6 microns was observed. Relating all the data to the common calibration plate, eliminates the thermal drift and mechanical settling therefore the repeatability error observed was mostly caused by the kinematic ball to wear pad mating repeatability. 


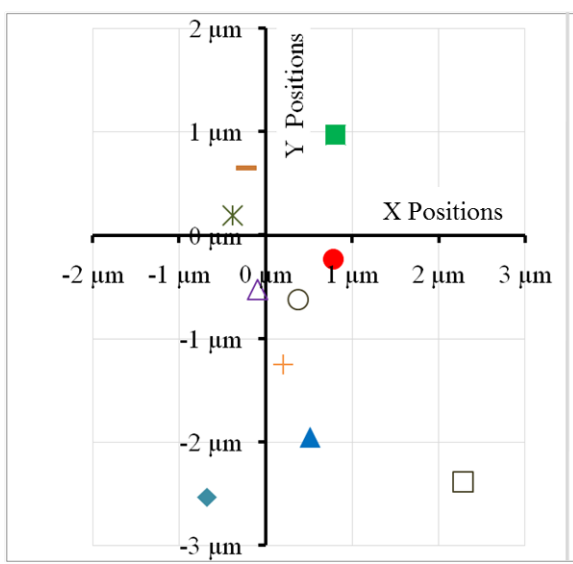

(a)

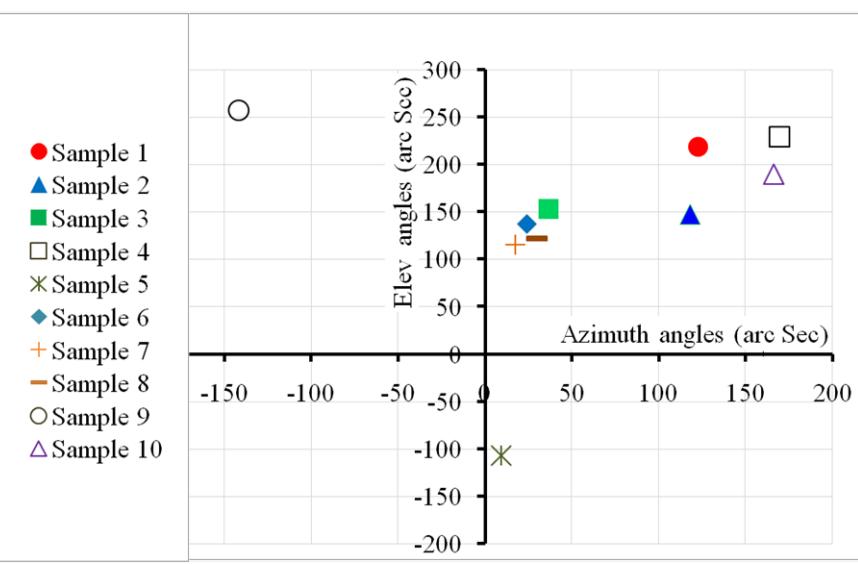

(b)

Figure 9. Experimental results of the apex X-Y position and the tilt angle. (a) Apex X-Y positions; (b) Plano surface Azimuth and Elevation angles.

\section{Summary}

We have demonstrated an optomechanical design and implementation for precision lens positioning and mounting metrology system. The performance of the metrology system, i.e. the lens's apex position and its plano surface tilt angle relative to OA of the instrument, was characterized. The accuracy of apex position of $\pm 3 \mu \mathrm{m}$ and the plano-surface tilt angle of $\pm 0.1^{\circ}$ was achieved. The precision of the metrology system for the lens mounting can be further improved by redesigning the kinematic-ball wear pad and replacing UV epoxy by laser welding for parts attachment. The capability to separate X-Y from tip-tilt alignment simplifies the alignment process for mass production. The design idea can be adapted to any application where $\mathrm{X}-\mathrm{Y}$ movement needs to be separated from the tip-tilt movement.

\section{References}

[1] Vukobratovich, D. (1999) Modular Optical Alignment. Proc. SPIE, 3786, 427-438. http://dx.doi.org/10.1117/12.363823

[2] Yoder, P. (2004) Optiomechanical Design in Five Easy Lessons. SPIE Oemagazine, 29.

[3] Wang, S., Zhang, C., Davis, C., Alt, M., Ji, Z., Han, Y. and Gardner, M.L. (2014) Optical Metrology Techniques and Apparatus for Lens Assembly. Proc. SPIE, 9272, 92720O. http://dx.doi.org/10.1117/12.2069606

\section{Scientific Research Publishing}

\section{Submit or recommend next manuscript to SCIRP and we will provide best service for you:}

Accepting pre-submission inquiries through Email, Facebook, LinkedIn, Twitter, etc. A wide selection of journals (inclusive of 9 subjects, more than 200 journals)

Providing 24-hour high-quality service

User-friendly online submission system

Fair and swift peer-review system

Efficient typesetting and proofreading procedure

Display of the result of downloads and visits, as well as the number of cited articles

Maximum dissemination of your research work

Submit your manuscript at: http://papersubmission.scirp.org/ 